\title{
Immunohistochemical detection of metalloproteinase-9 (MMP-9), anti-oxidant like 1 protein (AOP-1) and synaptosomal-associated protein (SNAP-25) in the cerebella of dogs naturally infected with spontaneous canine distemper
}

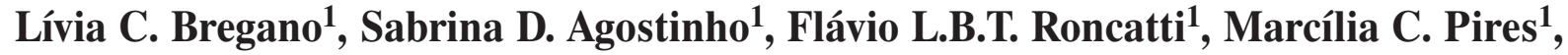 \\ Henrique G. Riva1, Maria C.R. Luvizotto ${ }^{1,2}$, Tereza C. Cardoso ${ }^{1}$ \\ ${ }^{1}$ Laboratory of Virology, Faculdade de Medicina Veterinária, campus Araçatuba, São Paulo, Brazil \\ ${ }^{2}$ Laboratory of Animal Pathology, Faculdade de Medicina Veterinária, campus Araçatuba, \\ São Paulo, Brazil
}

\begin{abstract}
In most viral infections of the central nervous system (CNS), the integrity of brain extracelluar matrix (ECM), oxidative stress and dysfunction in neuronal transmission may contribute to the observed pathology. The purpose of this study was to investigate the role of these factors in demyelinating canine distemper virus (CDV) infections. Regardless of ECM integrity, the expression of metalloproteinase-9 (MMP-9) was visualized in microglial-like cells, whereas the expression of anti-oxidant like-1 (AOP-1) and synaptosomal associated protein (SNAP-25) was frequently detected in Purkinje cells $\left(r^{2}=0.989 ; p<0.05\right)$, regardless of whether the lesions were classified as acute or chronic. Increased numbers of immunolabeled microglia-like cells and reactive gliosis were observed in advanced cases of demyelinating CDV, suggesting that the expression of AOP-1 and SNAP-25 is correlated with the ultimate death of affected cells. Our findings bring a new perspective to understanding the role of the AOP-1, MMP-9 and SNAP-25 proteins in mediating chronic leukoencephalitis caused by CDV. (Folia Histochemica et Cytobiologica 2011; Vol. 49, No. 1, pp. 41-48)
\end{abstract}

Key words: CDV, antioxidative stress, protein expression

\section{Introduction}

Infection with canine distemper virus (CDV), a single-stranded, negative-sense Morbillivirus of the family Paramyxoviridae, frequently affects the central nervous system (CNS) [1]. Demyelinating distemper leukoencephalitis (DL) represents the primary out-

Correspondence address: T.C. Cardoso, Departamento de Apoio, Produção e Saúde Animal, Curso de

Medicina Veterinária Rua Clóvis Pestana, 793.

16.050-680 Araçatuba, São Paulo, Brazil;

fax: (+ 55 18) 62264 87;

e-mail: tcardoso@fmva.unesp.br come in dogs. Due to the morphology of the neuropathological changes, this disease has come to be regarded as an important spontaneously occurring animal model of human demyelinating disease that is analogous to multiple sclerosis (MS) [2-4]. Initiation of demyelination has been described as a direct viral action, during which plaque progression seems to be mediated by this interaction [4]. During the initiation phase, viral proteins, mRNAs, major histocompatibility complex class II, hyaluronate receptor CD44 and pro-inflammatory cytokines are increased [1-7]. In contrast, chronic lesions are characterized by the reduction or loss of viral proteins and mRNA, a strong MHC II upregulation and a massive influx of monocytes [5]. 
Brain extracelluar matrix (ECM) plays a complex role in CNS maintenance and function [6]. The two major systems that modify the ECM in the CNS are matrix metalloproteinases (MMPs) and plasminogen activators (PAs) [6]. MMPs are zinc-dependent proteases that are secreted by most cell types as proenzymes. MMPs are catagorized in terms of their substrate specificity into classes such as gelatinases, collagenases and stromeolysins [6, 8]. MMPs play key roles in inflammatory responses through proteolytic release of cell membrane-bound factors and activation or inactivation of cytokines, chemokines and growth factors [9]. MMP-9 is known to digest gelatins and types III, IV and V collagen. Moreover, findings support a central role of MMP-9 in T-cell migration and in the disruption of the vascular basement membrane, and this protein has recently been shown to act directly in the blood-brain barrier after a virus infection [9-13]. Likewise, it has been demonstrated that a proportional increase in MMP mRNA-expressing cells might implicate an MMP imbalance as a cause for lesion initiation and progression in demyelinating canine distemper leukoencephalitis [11].

So, an altered balance between matrix metalloproteinases and their inhibitors seems to play an important role in the pathogenesis of chronic CDV infections [10].

The mitochondria play an important role in the aerobic energy metabolism of living cells [14]. The mitochondrial electron transport system consumes approximately $85 \%$ of the oxygen utilized by the cell, and about $5 \%$ of the oxygen is converted to reactive oxygen species (ROS). In the normal state of the cell, adequate concentrations of ROS participate in a wide variety of cellular functions, including cell proliferation, differentiation and apoptosis $[14,15]$. However, the significant induction of ROS, or the depletion of cellular antioxidants, induces cell death, and ROS are likely to act as signaling intermediates that are involved in the signal transduction mechanism of apoptosis [14]. Antioxidant-like protein-1 (AOP-1) is an antioxidant protein that functions as a thioredoxin-dependent peroxidase that scavenges ROS such as $\mathrm{H}_{2} \mathrm{O}_{2}$ [15]. AOP-1 expression is commonly detected in cases of transient forebrain ischemia, and is primarily found in non-pyramidal and astrocytic cells [15].

The synaptosomal-associated protein of $25 \mathrm{kDa}$ (SNAP-25) belongs to a superfamily of small membrane proteins that participate in the regulation of synaptic vesicle exocitosis [16]. Generation of SNAP-25 null mutant mice revealed that SNAP-25 is not required for stimulus-independent neurotransmitter release, but is essential for evoking synaptic transmis- sion [17]. SNAP-25 represents a multifunctional protein involved in the control of neurotransmitter secretion via several interactions. Recently, inter-cellular junctions have been shown to promote egress in alternate viral system, and CDV may use the gap junctions, which are indeed concentrated at the tip process, to spread into the astrocytic syncytial network [4]. However, how the viral fusion complex and its receptor interact with those cells remains unclear.

Little information is available about the interaction between AOP-1 and the dysfunction of synapses related to natural cases of CDV. In addition, there is a necessity to understand other mechanisms that can trigger CNS damage.

To this end, we aimed to further investigate the CDV-induced brain lesions associated with selected pathogenic events, such as the expression of anti-oxidant protein-1 (AOP-1) and synaptosome-associated protein (SNAP-25) as well as the status of the bloodbrain barrier integrity (MMP-9) in cerebella collected from 43 dogs afflicted with natural CDV.

\section{Material and methods}

\section{Sample collection and general procedures}

The cerebella of $20 \mathrm{CDV}$-negative, healthy control dogs (group I) and 43 dogs with spontaneous, molecularly confirmed, distemper leukoencephalitis (groups II-V) were examined. The dogs with distemper (20 males and 23 females between three months and 7 years of age), were animals submitted to the routine diagnostic service of the Laboratory of Animal Pathology, Veterinary College, University of São Paulo State, Araçatuba, Brazil. The animals died spontaneously or were killed for humane reasons, and all were considered negative for rabies co-infection.

For the present study, tissues including the CNS were collected, fixed in 10\% non-buffered formalin and embedded in paraffin wax, and serial sections of $4-\mu \mathrm{m}$ thickness were prepared. Serial sections for immunohistology were mounted on glass slides. Neuropathological diagnoses were performed for hematoxylin and eosin (HE)- and Shorr-stained sections (Sigma-Aldrich, St. Louis, MO, USA) [9]. In addition, detection of CDV proteins by immunofluorescence assay (IFA) was performed using a polyclonal antibody labeled with biotin (Biovet ${ }^{\circledR}$ Laboratories, Campinas, Brazil).

Distemper white matter lesions in HE-stained sections were classified into different groups as follows: group II (non-visible white matter lesions in HE-stained slides, i.e. no pathological changes on the HE-stained sections, but CDV positive cells); group 
III (acute lesions, i.e. focal vacuolization, mild gliosis with activated microglia and astrocytes, as well as cytoplasmatic and intranuclear inclusions bodies visualized by Shorr staining); group IV (subacute inflammatory lesions, i.e. demyelination, moderate gliosis with gemistocytes and multinucleated astrocytes, malacia and perivascular mononuclear infiltration of two or three layers of thickness); and group V (chronic lesions, i.e. more prominent perivascular infiltration of at least three layers of thickness) [9]. Because all four variants can occur in a single brain, the final neuropathological diagnosis was based on the most advanced type of white matter lesion in each animal.

\section{Immunofluorescence assay (IFA)}

For IFA, unstained histological sections were deparaffinized, rehydrated and washed in buffered saline with $0.1 \%$ Tween 80 (Sigma-Aldrich, St. Louis, MO, USA). Next, slides were washed three times for ten minutes in phosphate buffered saline (PBS) and blocked with $15 \%$ reconstituted dry non-fat milk for 90 minutes. The polyclonal antibody against CDV (Onderstepoort CDV strain) was kindly provided by Biovet ${ }^{\circledR}$ Laboratories (São Paulo, Brazil). The $\gamma$-globulin fraction was prepared by a salting-out procedure involving the addition of $35 \%(\mathrm{v} / \mathrm{v})$ of ammonium sulfate (A-2939, Sigma-Aldrich, St. Louis, MO, USA) and was followed by $\mathrm{IgG}$ fraction purification using chromatographic separation on Sephadex-G200 (GE Healthcare ${ }^{\circledR}$, Uppsala, Sweden). The purified IgG was conjugated to biotin (Biotin disulfide N-hydroxysuccinide ester, Sigma-Aldrich, St. Louis, MO, USA) and used as the primary antibody for CDV detection. The slides were covered with $100 \mu$ l of a 1:50 serum dilution and incubated overnight at $4^{\circ} \mathrm{C}$ in a humidified chamber. The secondary antibody was StrepAvidin-FITC (Sigma-Aldrich, St. Louis, MO, USA) and was capable of labeling the biotin present in viral particles positively detected by the antiserum. After one hour of incubation at $37^{\circ} \mathrm{C}$, the sections were covered by Fluoromount ${ }^{\circledR}$ (Sigma-Aldrich, St. Louis, MO, USA) and submitted to ultraviolet reflection light in an Axio Imager A.1 microscope (Carl Zeiss, Oberkochen, Germany).

\section{Immunohistochemistry assay (IHC)}

To perform the IHC assay, a standard avidin-biotinperoxidase complex (ABC, DakoCytomation, Carpentaria, CA, USA) method was used as described previously [9]. Unstained sections $(4 \mu \mathrm{m})$ were deparaffinized, rehydrated and washed in buffered saline with $0.1 \%$ Tween 80 . Sections were first microwaved in citrate buffer ( $\mathrm{pH}$ 6.1) for 15 minutes at 700 W to activate the GFAP (glial fibrillary acid protein), MMP-9 and AOP-1 antigens. The slides used for SNAP-25 immunodetection were not submitted to antigen reactivation. Just before staining, the slides were treated three times with $50 \%$ hydrogen peroxide (30 vol.) for 30 minutes to inactivate the endogenous peroxidase commonly found in inflammatory reactions. The slides were then washed five times for ten minutes each wash in buffered saline to remove the residue. Next, nonspecific binding was blocked by incubating with $15 \%$ reconstituted dry non-fat milk for 90 minutes. The optimum primary antibody dilutions used are provided in Table 1 . The slides were covered with $200 \mu \mathrm{l}$ of diluted antibody overnight at $4^{\circ} \mathrm{C}$ in a humidified chamber. After five washes, 100 $\mu l$ of streptavidin-peroxidase complex (Dako Cytomation, Carpentaria, CA, USA) was added to each slide, and the slides were incubated for one hour at $37^{\circ} \mathrm{C}$. In addition, a substrate AEC staining kit and liquid DAB was used following the manufacturer's instructions (Sigma-Aldrich, St. Louis, MO, USA). The reaction was stopped by washing with tap water, and a specific brown and red color was revealed after counterstaining with aqueous Hematoxylin (Sigma-Aldrich, St. Louis, MO, USA). An intense brownish-red deposit was considered positive. Omission of the primary antibody was used as a negative control for the different antibodies. A canine mammary carcinoma histological section was used as an MMP-9 positive control.

\section{Scoring and statistical analysis}

Differences among the five groups (I-V) and the total of immunoreactive cells (calculated using a morphometric grid; number of cells $/ \mathrm{mm}^{2}$ ) to MMP-9,

Table 1. Specifications of the primary antibodies used

\begin{tabular}{|l|c|c|c|c|}
\hline & Antibody & Dilution & Species & Supplier \\
\hline AOP-1 & Anti-oxidant like protein-1 & $1: 1,000$ & Mouse & Sigma-Aldrich ${ }^{\circledR}$ \\
\hline GFAP & Glial fribrillary acidic protein & $1: 100$ & Mouse & Dako® \\
\hline MMP-9 & Metalloproteinase 9 & $1: 200$ & Mouse & Calbiochem ${ }^{\circledR}$ \\
\hline SNAP-25 & Synaptosomal-associated 25kDa protein & $1: 10,000$ & Mouse & Prestige Antibodies $^{\mathrm{TM}}$ \\
\hline
\end{tabular}


SNAP-25 and AOP-1 were tested using unpaired Student's $t$-test preceded by evaluation of normality and Levene's test. Correlation coefficient was calculated using Spearman's method for immunoreactivity of stained cells and cell type (neurons, microglial cells; astrocytes and Purkinje cells). Additionally, to evaluate MMPs staining, the intensity of the color was scored as follows: - , no staining; + , slight staining intensity; ++ , moderate staining intensity; +++ , marked staining intensity. The number of positively infected cells was estimated using a light Axio Imager A.1 microscope connected to an AxioCam MRc (Carl Zeiss). The micrographs were processed with AxioVision 4.7 software (Carl Zeiss). SAS v. 8.2 (SAS Institute Inc. Cary, NC, USA) and Origin software was used for data analysis. Results were considered statistically significant if $\mathrm{p}<0.05$.

\section{Ethical concerns}

All animal handling and sample collection procedures were performed in accordance with the recommendations of the Brazilian College on Animal Experimentation (COBEA), and all experiments were approved by the Institution of Ethics and Animal Welfare Committee.

\section{Results}

\section{Neuropathological findings}

Cerebellar tissues from healthy control dogs $(n=20)$ showed no pathological alterations (group I, randomly selected white matter and granular layer areas; $n=56$; total area for each dog: $1 \mathrm{~mm}^{2}$ ). A total of 100 brain lesions were investigated and classified into groups II-V (II, $\mathrm{n}=25 ; \mathrm{III}, \mathrm{n}=25 ; \mathrm{IV}, \mathrm{n}=25 ; \mathrm{V}, \mathrm{n}=25$ ) in 43 distemper dogs. The overall neuropathological diagnoses revealed that ten dogs showed acute encephalitis, ten dogs displayed sub-acute inflammatory distemper leukoencephalitis, and 23 dogs were suffering from the chronic stages of distemper leukoencephalitis. Figure 1 (A-D) shows representative cerebellar histological sections from groups II-IV. As shown, an influx of inflammatory cells and initial vacuolization were primarily observed among animals in groups IV and V (Figures $1 \mathrm{C}$ and D).

\section{Quantitative comparison of IHC findings of MMP-9, SNAP-25 and AOP-1}

Comparisons of the groups (I-V) and the total of immunoreactive cells, independent of their type, to MMP-9, SNAP-25 and AOP-1 expression are de- scribed in Figure 2. The mean values of CDV-positive cells staining and GFAP-immunoreactive astrocytes were only seen in group III. However, groups IV and $\mathrm{V}$ presented the same pattern of immunolabel for MMP-9, AOP-1 and SNAP-25, and only group IV showed GFAP-immunoreactive astrocytes when compared to group V. In the control group, MMP-9, AOP-1 and SNAP-25 antigens were not detectable (Figure 2). In contrast to the lower intensity of MMP-9, SNAP-25 and AOP-1 among dogs belonging to group II $\left(<20 \%\right.$-immunoreactive cells $\left./ \mathrm{mm}^{2}\right)$, a positive correlation coefficient was obtained from the values computed from groups III, IV and V ( $>60 \%$ immunoreactive cells $\left./ \mathrm{mm}^{2} ; \mathrm{r}^{2}=0.989 ; \mathrm{p}<0.05\right)$.

\section{Qualitative comparison of findings of MMP-9, SNAP-25 and AOP-1}

The CDV antigens were frequently visualized in astrocytes and oligondendrocytes-like cells from group III and less frequently detected in groups II, IV and V (Table 2). In distemper dogs of groups II and III, the number of GFAP-positive astrocytes was scored as marked staining intensity representing astrogliosis and astrocytosis phenomena (Figures 3A and B). The expression and distribution pattern of SNAP-25 is shown in Figures 4A and B. The majority of the labeled cells were identified as being Purkinje cells, where a brownish cytoplasm was clearly evident. In the white matter, a diffuse SNAP-25 label was primarily visualized surrounding neurons (Figure 4B). Moreover, a marked staining intensity in the cytoplasm of microglial-like cells to MMP-9 antigens was observed in groups III through $\mathrm{V}$ as demonstrated by the red deposits (Figure 4C). In addition, the granular layer showed moderate staining intensity of AOP-1 labeled cells (Purkinje cells) was observed in groups III through V (Figure $4 \mathrm{D})$. The control group $\left(\mathrm{CDV}^{-}\right)$ruled out nonspecific reactions to any antibody use in this trial.

\section{Discussion}

In addition to the CNS diseases caused primarily by neurotropic viruses, neurological complications are associated with a number of viral infections. Some neurological complications following infection with Morbillivirus occur in the context of genetic or acquired immunodeficiency, while others are associated with extensive inflammatory response. In general, the histopathological changes observed in the cases of distemper disease studied in this investigation concord with previous reports worldwide $[1,2,8,9]$. Moreover, the microscopic aspects have not diverged from the literature, especially relative to the degene- 


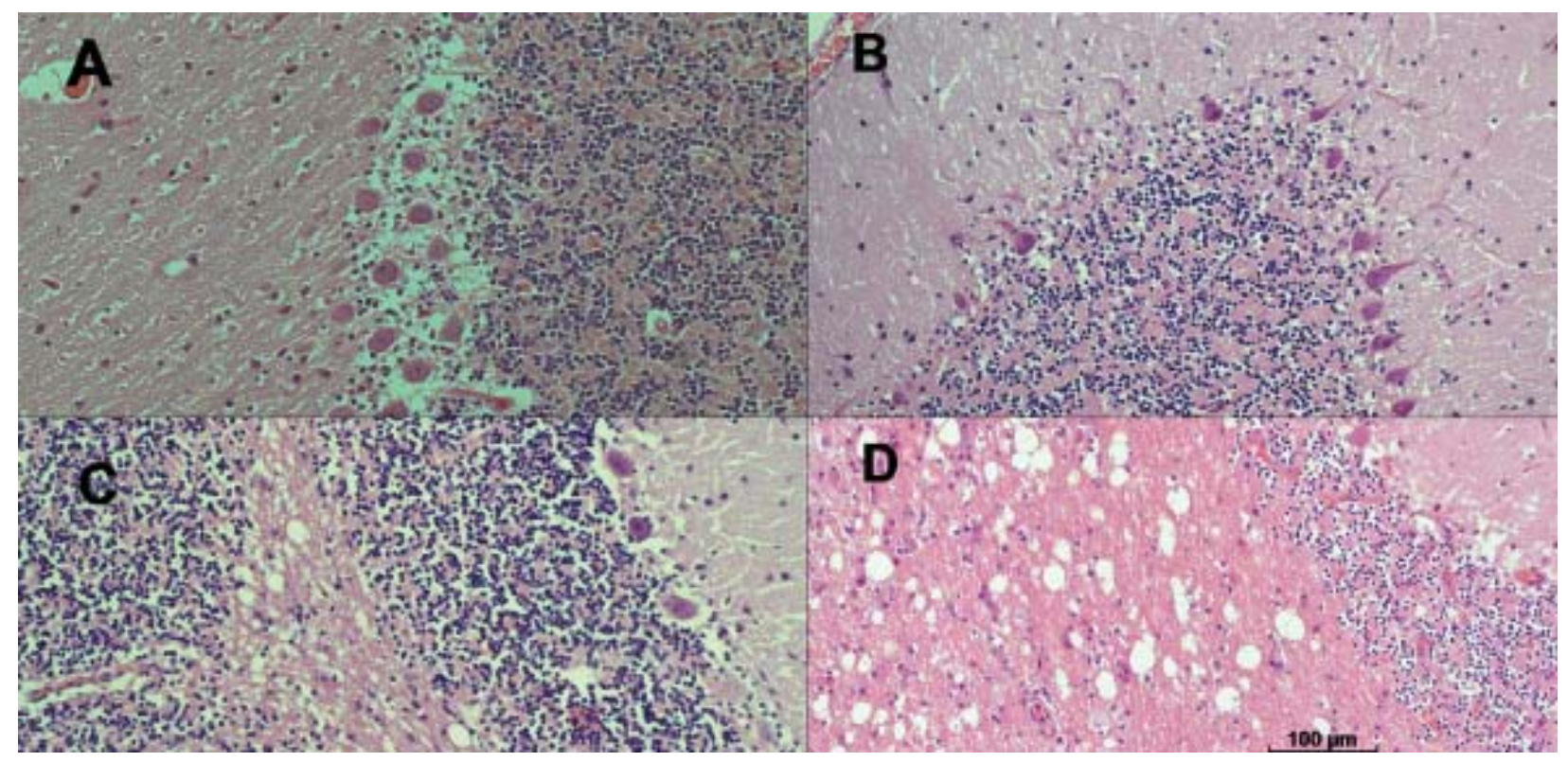

Figure 1. Photomicrography of microscopic aspects from CDV infected dogs' cerebella. A. Group II, no pathological changes on the cerebellum HE sections; B. Group III, inflammatory influx, moderate gliosis; C. Group IV, initial focal vacuolization; D. Group V; intense vacuolization associated with malacia (scale bar $100-\mu \mathrm{m}$; magnification $\times 40$ )

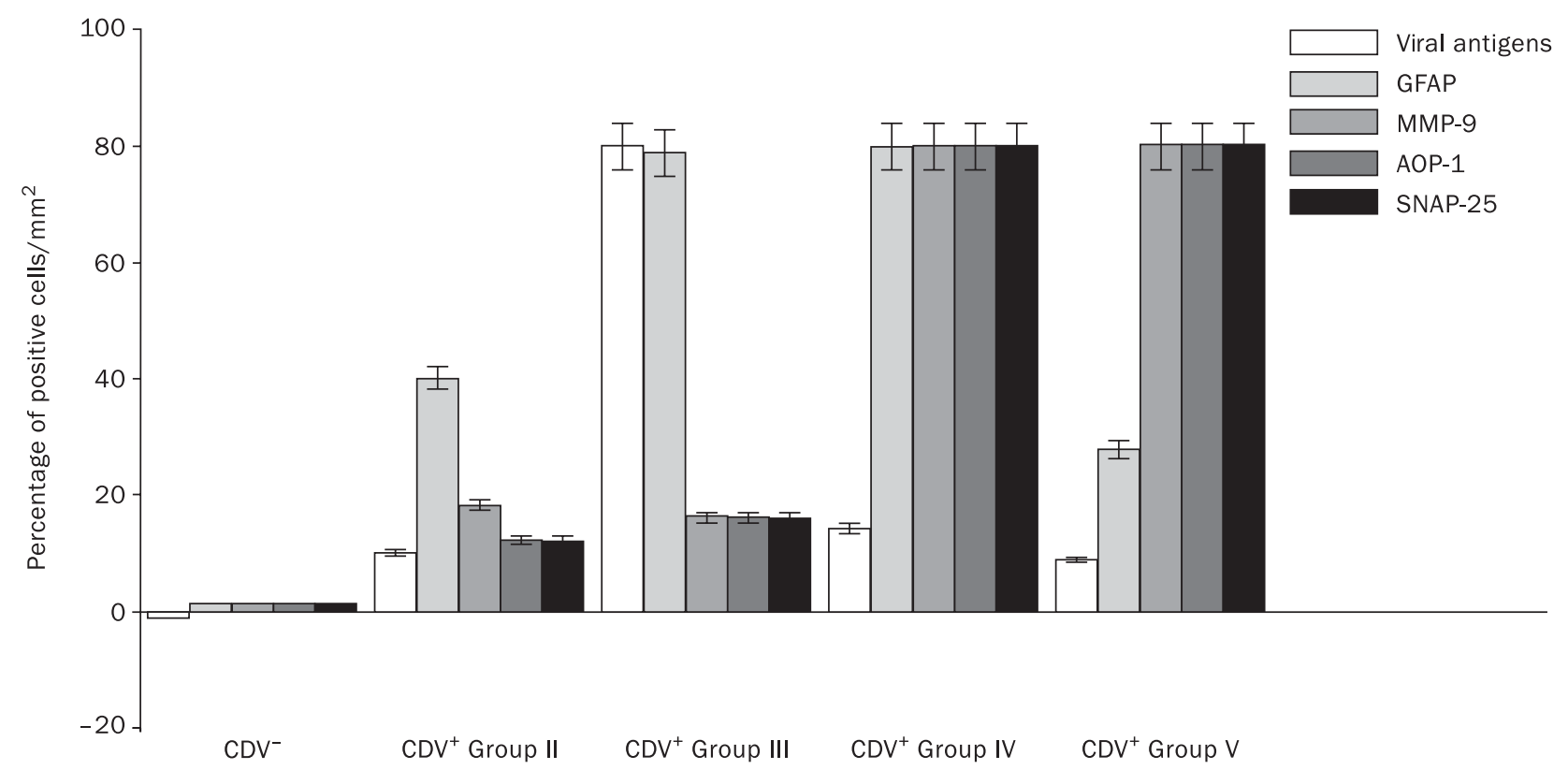

Figure 2. The frequency of detection of $\mathrm{CDV}^{-}$and $\mathrm{CDV}^{+}$antigens and AOP-1, MMP-9 and SNAP-25 proteins in the cerebella of dogs naturally infected with $\mathrm{CDV}$ and a control group including all labeled cells independent of cell type

ration process [4]. In the evolution to the chronic stage, the commonest histological finding was an intense vacuolization localized to areas of malacia. The influx of inflammatory cells associated with the initial stage of astrogliosis was detected in group II, while the CDV antigens were also detected. This phenomenon is well established in CDV studies and was reproduced by our analysis $[5,7]$.
Recently, it has been demonstrated that the inflammatory process induced by stimuli from the CNS leads to a primary astrocyte dysfunction resulting in a demyelination process [6]. On the other hand, upon generalized immunosuppression associated with CDV infections, little inflammation has been observed in the CNS of dogs during the acute phase of the disease [18]. However, immunohistochemical studies 
Table 2. Quantitative comparison of immunoreactive-cell types in all studied groups to SNAP-25, MMP-9 and AOP-1 antigens expressed as mean $\pm(\mathrm{SD})$

\begin{tabular}{|l|c|c|c|c|c|c|}
\hline \multicolumn{9}{|c|}{ Number and type of immunoreactive cells } \\
\hline & Group I (n = 25) & Group II (n= 25) & Group III (n= 25) & Group IV (n= 25) & Group V (n= 25) & p \\
\hline GFAP & ND & $8 \pm 1.7$ Astrocytes & $78 \pm 12.7$ Astrocytes & $88 \pm 14.3$ Astrocytes & $18 \pm 6.6$ Astrocytes & \multirow{2}{*}{0.989} \\
\hline SNAP-25 & ND & $3 \pm 0.7$ Purkinje cells & $18 \pm 7.7$ Purkinje cells & $79 \pm 11.7$ Purkinje cells & $88 \pm 12.3$ Purkinje cells \\
\hline MMP-9 & ND & $10 \pm 1.1$ Microglial & $16 \pm 5.6$ Microglial & $74 \pm 10.7$ Microglial & $72 \pm 11.7$ Microglial \\
\hline AOP-1 & ND & $2 \pm 0.7$ Purkinje cells & $15 \pm 3.7$ Purkinje cells & $88 \pm 13.7$ Purkinje cells & $86 \pm 14.7$ Purkinje cells & \\
\hline
\end{tabular}

ND - not detected

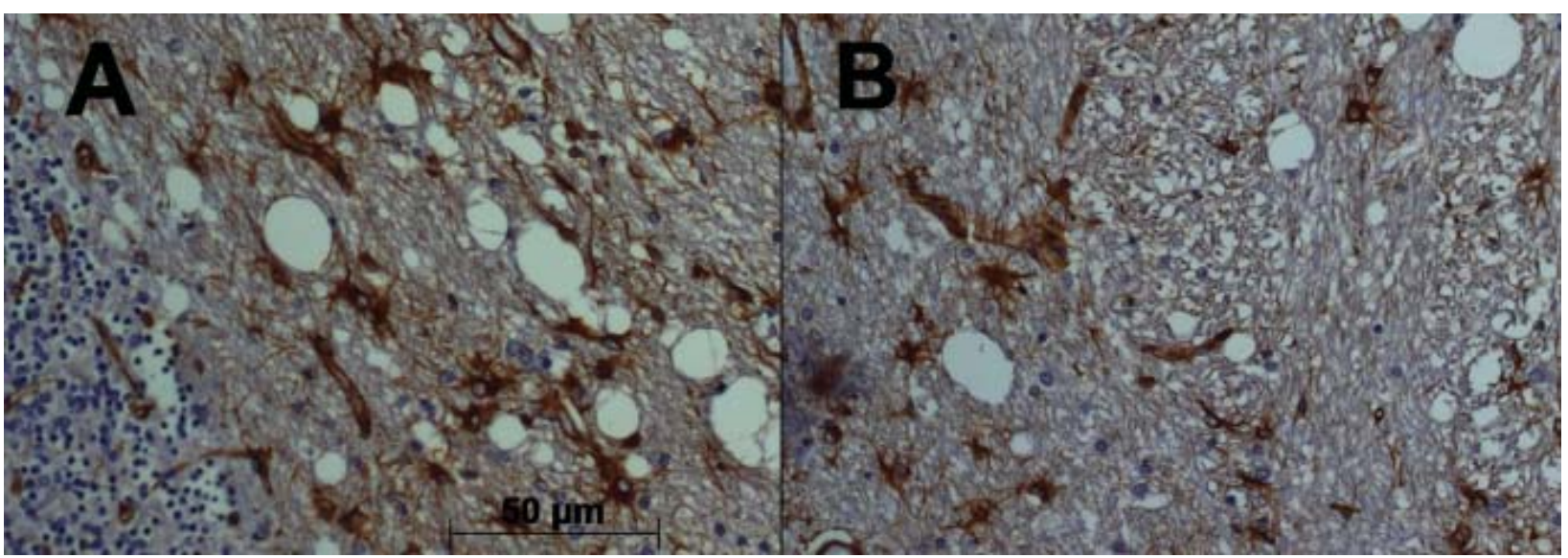

Figure 3. Photomicrography of GFAP-immunoreactive astrocytes detected by IHC in the cerebella of dogs naturally infected with CDV. A. Intense immunoreactions in the granular layer associated with the vacuolization process (group III; magnification $\times 20$ ); B. High magnification $\times 40$. The DAB complex method was used (scale bar $50 \mu \mathrm{m})$

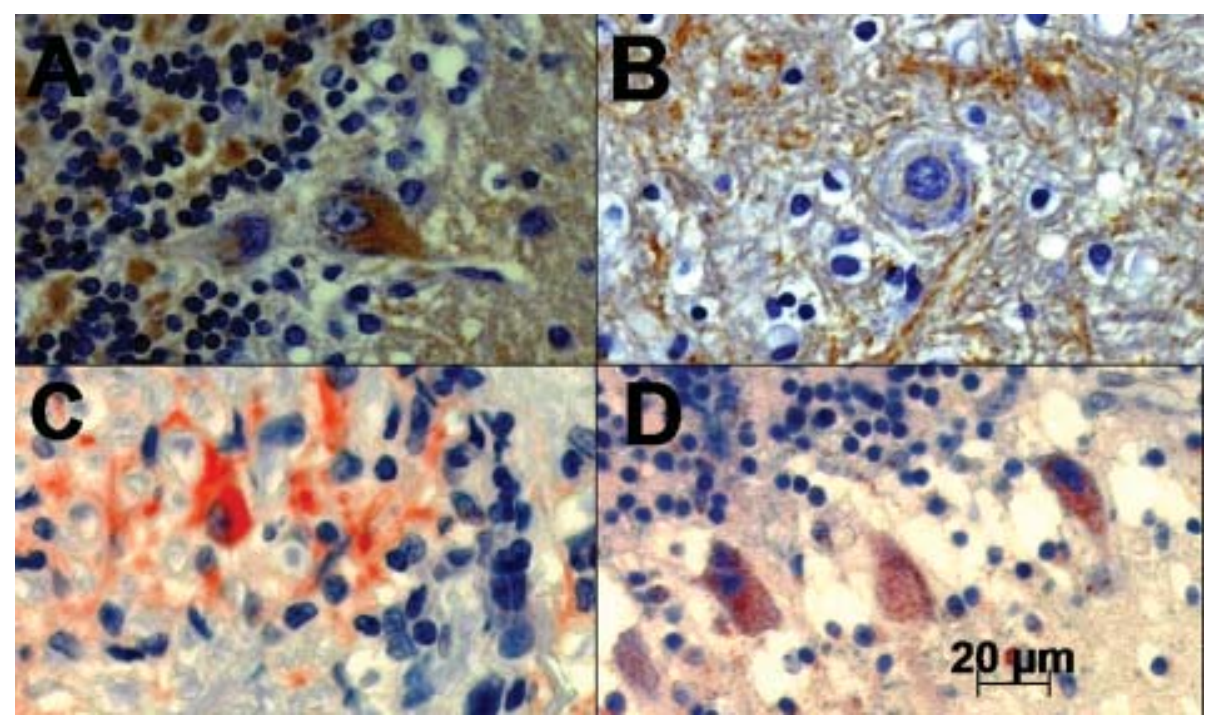

Figure 4. Photomicrography of SNAP-25, MMP-9 and AOP-1 antigens detected by IHC in the cerebella of dogs naturally infected with CDV. A. Intense SNAP-25 immunoreactivity in the cytoplasm of Purkinje cells; B. Intense SNAP-25 positive signals dispersed within the white matter surrounding the neuron; C. A positive cytoplasmatic reaction of MMP-9 in microglial-like cells; D. The granular layer showing positive AOP-1 in Purkinje cells. The DAB and AEC complex method was used, respectively (scale bar $20 \mu \mathrm{m}$; magnification $\times 40$ ) 
have revealed the presence of immune cells as early as the acute and subacute phases of non-inflammatory lesions. This suggests that this subdivision is a somewhat arbitrary approach to classifying distemper degeneration [2].

The remarkable result presented here is the association of the expression of MMP-9, AOP- 1 and SNAP-25 antigens during the progress of the chronic CDV stage. It would seem that MMPs play a crucial role in CDV lesion initiation in advanced infections [9]. It has also been demonstrated that MMP-9 facilitates the entry of West Nile Virus into the brain [19]. Their inhibitors, the TIMPs (tissue inhibitors of matrix metalloproteinases), are upregulated in acute and subacute non-inflammatory lesions and are expressed primarily by astrocytes, macrophages, and microglia (as previously demonstrated) [12]. In addition, it has been demonstrated that astrocytes play a key role in the activation of microglia in persistent Borna disease virus infection [20]. It has been demonstrated that the spread of measles virus depends on the cleavage of its envelope fusion protein by MMPs [21].

In fact, the integrity of the ECM may compromise the aerobic energy metabolism in nervous cells. This hypothesis is supported by the increase in AOP-1 expression in the granular layer in the advanced demyelinating process in this study. Recently, it was demonstrated that human Paramyxovirus infection alters mitochondrial metabolism [22]. The basic hypothesis for this investigation was that some viruses may play an important role in the etiology of disease in which energy generation is impaired [22]. Thus it has been shown that measles induces mitochondrial structural abnormalities in dendrites during the persistent infection of the brain known as subacute sclerosing panencephalitis (SSPE) [23]. In conclusion, it has been demonstrated that the spread of measles virus is dependent on the cleavage of the envelope fusion protein by MMPs and also interference with the mitochondrial metabolism [21]. How this oxidative stress could directly/indirectly interfere with the synapse process needs to be established.

In ferrets, CDV can also utilize neuronal pathways to enter and spread through the brain [4]. However, the infection of white matter astrocytes is specific to $\mathrm{CDV}$ in dogs, is relevant to the demyelinating process, and is the main target of CDV in vivo and in vitro [4]. The synaptic vesicle-associated proteins, synapsins, play different roles in the two types of neurons: glutamatergic and gabaergic neurons [23].

Growing evidence supports the hypothesis that SNAP-25 also modulates various voltage-gated ion channels and represents a protein involved in the neurotransmission process. Moreover, SNAP-25 is the most abundant protein in the brain, constituting $1 \%$ of brain proteins, and is not exclusively localized at synaptic sites but is also present along axons and dendrites [23]. In our study, SNAP-25 protein was clearly detected inside the cytoplasm, mostly in Purkinje cells. The proportional increase of AOP-1 and SNAP-25 in groups IV and V might indicate an imbalance of aerobic energy and neurotransmission. However, further investigations of this interaction are needed to elucidate the complex activation of the mitochondrial metabolism during CDV infections.

\section{Acknowledgments}

This project was supported by the Conselho Nacional Pesquisa (CNPq n $\left.{ }^{\circ} 472226 / 2007-0\right)$ and the Fundação Amparo à Pesquisa Estado de São Paulo (2008/03802-2). Tereza Cristina Cardoso is a recipient of CNPq council grants.

\section{References}

1. Griot C, Vandelvelde M, Schobesberger M, Zurbriggen A. Canine distemper, a re-emerging Morbillivirus with complex neuropathogenic mechanisms. Anim Health Res Rev. 2003; 4:1-10.

2. Beineke A, Seehusen F, Baumgärtner W. Pathogenesis and immunopathology of systemic and nervous canine distemper. Vet Immunol Immunopathol. 2009;127:1-18.

3. Schobesberger M, Summerfield A, Doherr MG, Zurbriggen A, Griot C. Canine distemper virus-induced depletion of uninfected lymphocytes is associated with apoptosis. Vet Immunol Immunopathol. 2005;104:3-44.

4. Wyss-Fluehmann G, Zurbriggen A, Vandevelde M, Plattet P. Canine distemper virus persistence in demyelinating encephalitis by swift intracellular cell-to-cell spread in astrocytes is controlled by viral attachment protein. Acta Neuropathol.2010;119:617-630.

5. Wünschman A, Kremmer E, Baumgärtner W. Phenotypical characterization of $\mathrm{T}$ and $\mathrm{B}$ cell areas in lymphoid tissues of dogs with spontaneous distemper. Vet Immunol Immunopathol. 2000;73:83-98.

6. Sharma R, Fisher M-T, Bauer J et al. Inflammation induced by innate immunity in the central nervous system leads to primary astrocytes dysfunction followed by demyelination. Acta Neuropathol. 2010;120:223-236.

7. Seehusen F, Orlando EA, Wewetzer K, Baumgärtner W. Vimentin-positive astrocytes in canine distemper: a target for canine distemper virus especially in chronic demyelinating lesions? Acta Neuropathol. 2007;114:597-608.

8. Candelario-Jalil E, Yang Y, Rosenberg GA. Diverse roles of matrix metalloproteinases and tissue inhibitors of metalloproteinases in neuroinflammation and cerebral ischemia. Neuroscience. 2009,158:983-994.

9. Gröters S, Alldinger S, Baumgärtner W. Up-regulation of mRNA for matrix metalloproteinases- 9 and -14 in advanced lesions of demyelinating canine distemper leukoencephalitis. Acta Neuropathol. 2005;110:369-382.

10. Leppert D, Waubant E, Galardy R, Bunnet NW, Hauser SL. $\mathrm{T}$ cell gelatinases mediate basement membrane transmigration in vitro. J Immunol. 1995;154:4379-4389. 
11. Saadoun D, Bieche I, Authier F-J et al. Role of matrix metalloproteinase, proinflammatory cytokines and oxidative stressderived molecules in Hepatitis C virus-associated mixed cryoglobulemia vasculitis neuropathy. Arthritis and Rheumatism. 2007;56:1315-1324.

12. Miao Q, Baumgärtner W, Failing K, Alldinger S. Phase-dependent expression of matrix metalloproteinases and their inhibitors in demyelinating canine distemper encephalitis. Acta Neuropathol. 2003;106:486-494.

13. van Lint P, Libert C. Chemokine and cytokine processing by matrix metalloproteinases and its effect on leukocyte migration and inflammation. J Leukoc. 2007;82:1375$-1381$.

14. Bras M, Queenan B, Susin AS. Programmed cell death via mitochondria: different modes of dying. Biochemistry. 2005;70:284-293.

15. Hwang IK, Hua L, Yoo Ki-Y et al. Anti-oxidant-like protein 1 is altered in non-pyramidal cells and expressed in astrocytes in gerbil hippocampal CA1 region after transient forebrain ischemia. Brain Res. 2005;1002:111-119.

16. Matteoli M, Pozzi D, Grumelli $G$ et al. The synaptic split of SNAP-25: different roles in glutamatergic and gabaergic neurons? Neuroscience. 2009;158:223-230.
17. Fellin T. Communication between neurons and astrocytes: relevance to the modulation of synaptic and network activity. J Neurochemistry. 2009;108:533-544.

18. Rudd PA, Bastien-Hamel L-E, Von Messling V. Acute canine distemper encephalitis is associated with rapid neuronal loss and local immune activation. J Gen Virol. 2010;91:980-989.

19. Wang P, Dai J, Bai F et al. Matrix metalloproteinase 9 facilitates West Nile Virus entry into the brain. $J$ Virology. 2006;82:8978-8985.

20. Ovanesov M, Ayhan Y, Wolbert C, Moldovan K, Sauder C, Pletnikov V. Astrocytes play a key role in activation of microglia by persistent Borna disease virus infection. J Neuroinflammation. 2008;5:doi:10.1186/1742-2094-5-50.

21. Paula-Barbosa MM, Tavares MA, Borges MM. Mitochondrial abnormalities in cortical dendrites from patients with early forms of subacute sclerosing panencephalitis (SSPE). Acta Neuropathol 1984;63:117-22.

22. Derakhshan M. Effect of measles virus (MV) on mitochondrial respiration. Indian J Med Res. 2010;131:97-100.

23. Springfeld C, von Messling V, Frenzke M, Ungerechts G, Buchholz CJ, Cattaneo R. Oncolytic efficacy and enhanced safety of measles virus activated by tumor-secreted matrix metalloproteinases. Cancer Res. 2006;66:7694-7700.

Submitted: 9 September, 2010 Accepted after reviews: 30 January, 2011 